\title{
Nonsyndromic Multiple Basal Cell Carcinomas
}

\author{
Dong Hwi Kim, \\ Hyo Sun Ko, \\ Young Joon Jun \\ Department of Plastic and Reconstructive \\ Surgery, Bucheon St. Mary's Hospital, College \\ of Medicine, The Catholic University of Korea, \\ Seoul, Korea
}

No potential conflict of interest relevant to this article was reported.

\begin{abstract}
Basal cell carcinoma (BCC) comprising several lesions is not uncommon, but nonsyndromic multiple BCCs with parotid invasion are rare entities. We present two cases of multiple sporadic, nonsyndromic BCCs, and one of these cases is a unique case of parotid invasion associated purely with actinic keratosis. In Case 1, a 79-year-old female presented with multiple skin lesions on the face and left hand. All lesions were completely removed by surgery. The pathologic results showed lesions consistent with $\mathrm{BCC}$ and some lesions consistent with actinic keratosis. After 8 months, the patient presented with skin lesions in bilateral temporal areas and left cheek area. Surgical excision of the lesions was performed, and the biopsy results were squamous cell carcinoma in situ and actinic keratosis. In Case 2, a 43-year-old woman presented with multiple skin lesions on the face, scalp, right chest, abdomen and right leg. All lesions were completely removed by surgery. Pathologic evaluation confirmed the diagnosis of BCC. BCC is rarely metastatic, but it can lead to severe disfiguration or destruction. It is important to diagnose and treat $\mathrm{BCC}$ at an early stage.
\end{abstract}

Keywords: Basal cell carcinoma, nonsyndromic / Basal cell carcinoma, multiple / Skin neoplasms

\section{INTRODUCTION}

Basal cell carcinoma (BCC) is the most common form of skin cancer with multifactorial causes. Ultraviolet radiation exposure and genetic predisposition are known to be the most significant etiological factors along with other factors including Fitzpatrick skin types I and II, increasing age, male sex, arsenic and dry ice exposure and a high dietary fat intake [1,2]. Multiple BCCs are often related to heredofamilial conditions like nevoid BCC syndrome (Gorlin syndrome), Rombo syndrome, Bazex syndrome, and unilateral basal cell nevus syndrome [3,4]. BCC comprising several lesions is not uncommon, but nonsyndromic multiple BCCs with parotid invasion are rare entities. We present two cases of multiple sporadic, nonsyndromic BCCs, and one of these cases is a unique case of parotid invasion associated purely with actinic keratosis.

\section{Correspondence: Young Joon Jun}

Department of Plastic and Reconstructive Surgery, Bucheon St. Mary's Hospital, College of Medicine, The Catholic University of Korea, 327 Sosa-ro, Wonmi-gu, Bucheon 14647, Korea

E-mail: joony@catholic.ac.kr

Received June 13, 2017 / Revised September 8, 2017 / Accepted September 8, 2017

\section{CASE REPORT}

In Case 1, a 79-year-old Korean female patient with Fitzpatrick skin type III visited our institution with a complaint of multiple, variable sized skin lesions with itching sensation on the face and the left hand. The lesions were located on bilateral posterior auricular areas, forehead, right cheek, left temple and left hand, and they could be classified into nodular, superficial, pigmented and morphoeic variants and had gradually increased in size over approximately 10 years (Fig. 1). The patient had previously received surgical excision for BCC on the right posterior auricular area at another hospital 7 years ago. Also 1 year ago, she had received surgical excision for a single BCC lesion on the lower lip. The patient was engaged in agriculture and was constantly exposed to direct sunlight for most of her lifetime. She denied any family history of skin cancer, past history of cryotherapy or radiotherapy, exposure to chemical substances including arsenic, dry ice, and she showed no regional lymphadenopathy. The whole body bone scan for systemic metastases revealed no distant metastasis and punch biopsies depicted findings consistent with Bowenoid 

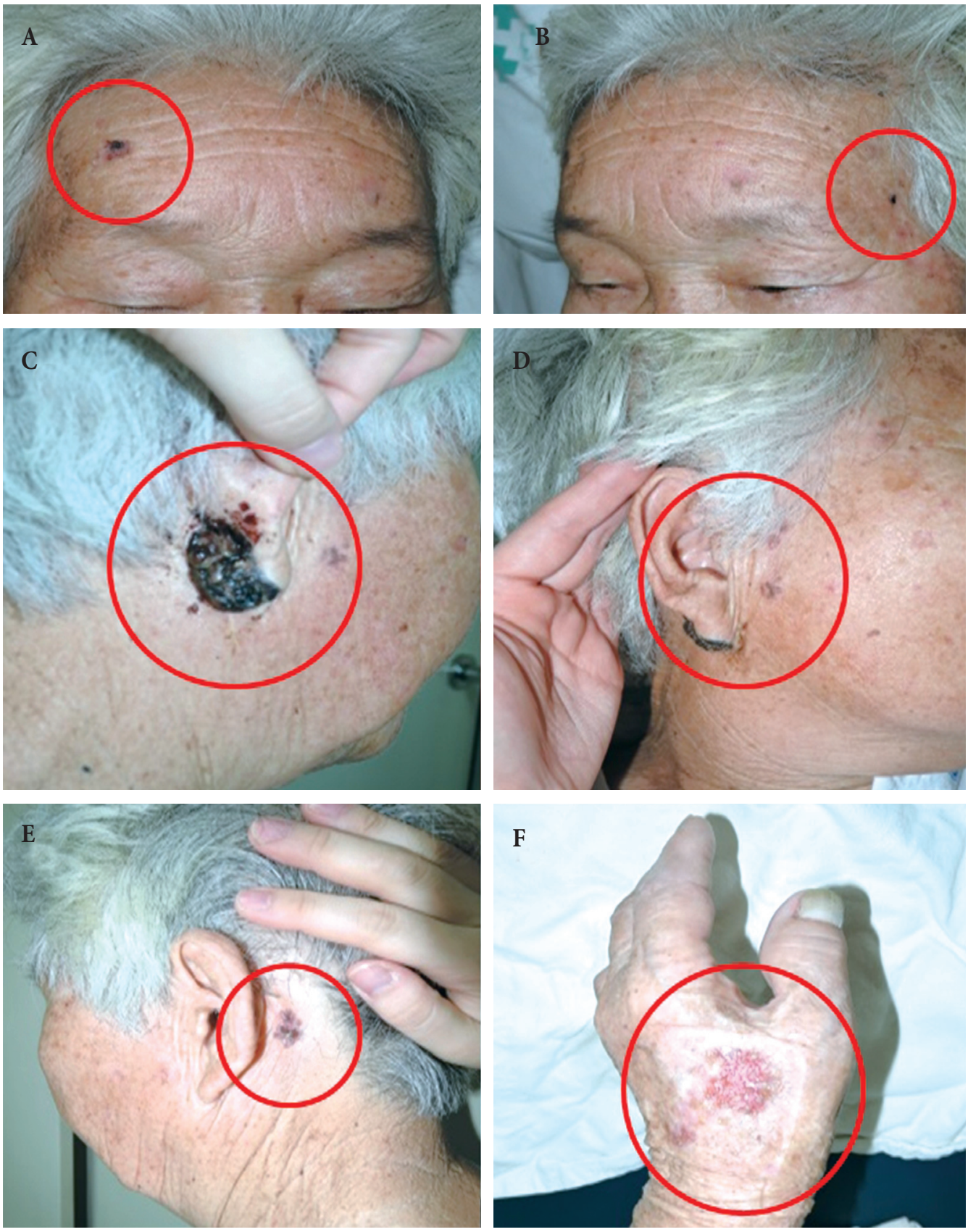

Fig. 1. Case 1. Preoperative photography shows that the lesions (red circles) were located on (A) forehead, (B) left temple (C, D, E) posterior auricular areas, (D) right cheek, and (F) left hand.

actinic keratosis. The lesion in the right posterior auricular area was particularly large in size and magnetic resonance imaging scan showed that it was in direct contact with the parotid gland (Fig. 2). All tumors were completely removed with Mohs micro- graphic surgery (MMS), except for the lesion in the right posterior auricular area, for which wide excision with partial parotidectomy using a fasciocutaneous advancement flap was employed (Fig. 3), as BCCs located in the periauricular embryonic fusion 

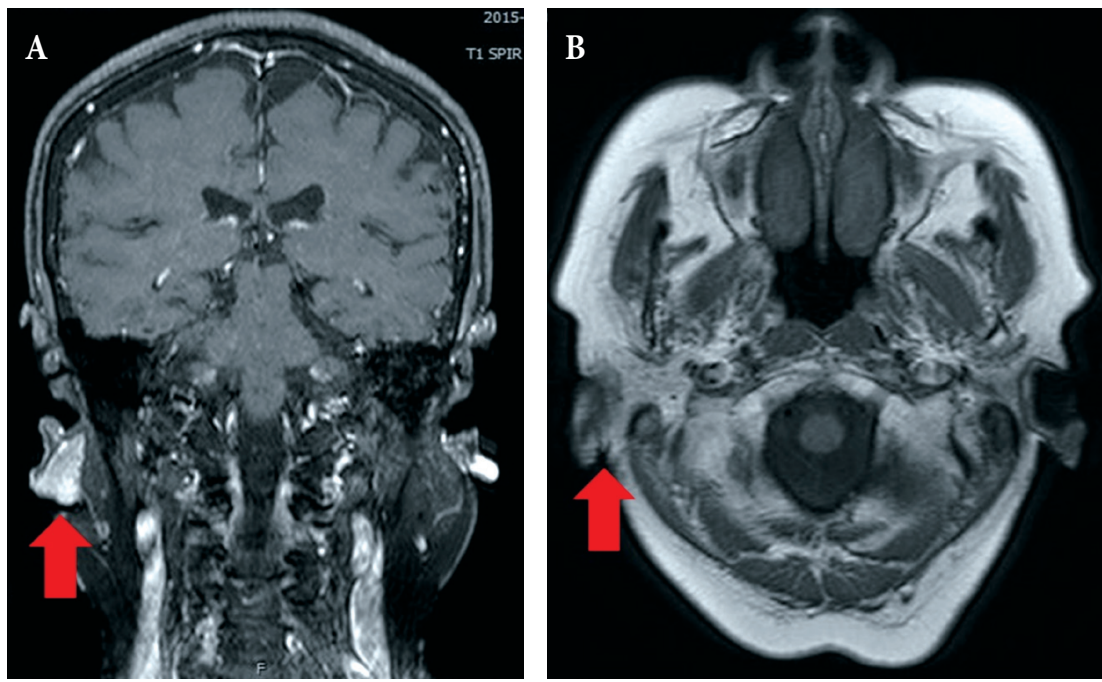

Fig. 2. Case 1. (A, B) Facial magnetic resonance imaging showing that the right posterior auricular basal cell carcinoma (red arrows) was in direct contact with the parotid gland.
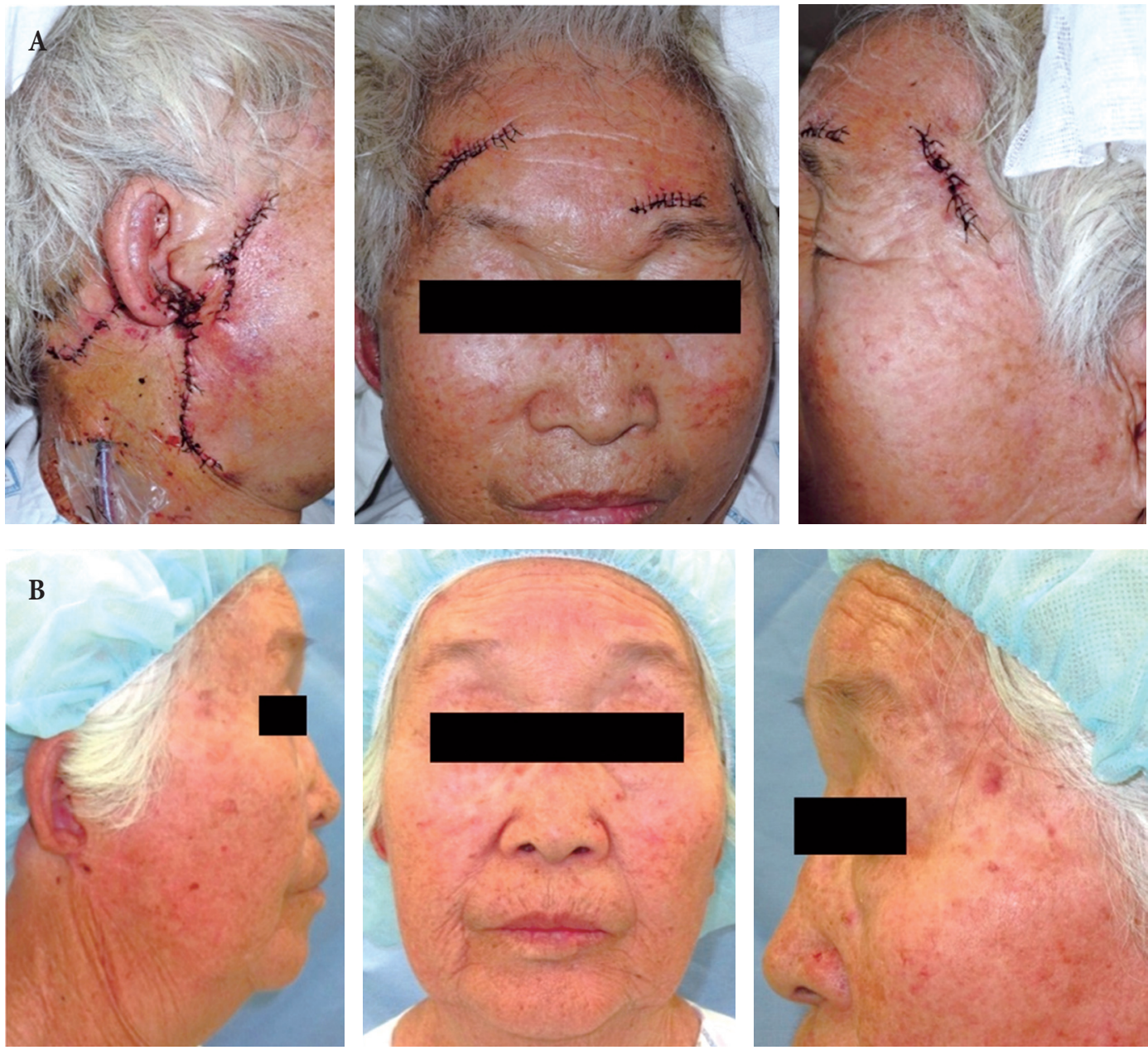

Fig. 3. Case 1. (A) Immediate postoperative clinical photograph. (B) A 7-month follow-up photograph. 
planes have an increased risk of metastasis [5]. The pathologic results showed lesions consistent with BCC and some lesions consistent with actinic keratosis. Strands of BCC are directly adhered to the parotid gland, and extend to the parotid tissue located at the left bottom of the photograph (Fig. 4). In cases of recurrence of BCC in pre-auricular or postauricular skin, it is very likely that the lesion has invaded the parotid gland [6]. To make diagnostic confirm for parotid invasion, the patient was consulted by the specialists of multidisciplinary care system and clinically confirmed as parotid invasion of BCC. After 8 months, the patient complained of skin lesions in bilateral temporal areas and left cheek area. Surgical excision of the lesions was performed to obtain negative margins, and the biopsy results were squamous cell carcinoma in situ and actinic keratosis. During the 18-month follow-up period, there were no complications or recurrences.

In Case 2, a 43-year-old Korean woman visited our institution with a complaint of multiple, variable sized skin lesions with itching sensation on the face, scalp, right chest, abdomen and right leg (Fig. 5). The lesions could be classified into nodular, superficial, pigmented and morphoeic variants. She denied any family history of skin cancer, past history of cryotherapy or radiotherapy, exposure to chemical substances including arsenic, dry ice, and she showed no regional lymphadenopathy. All tumors were complete-

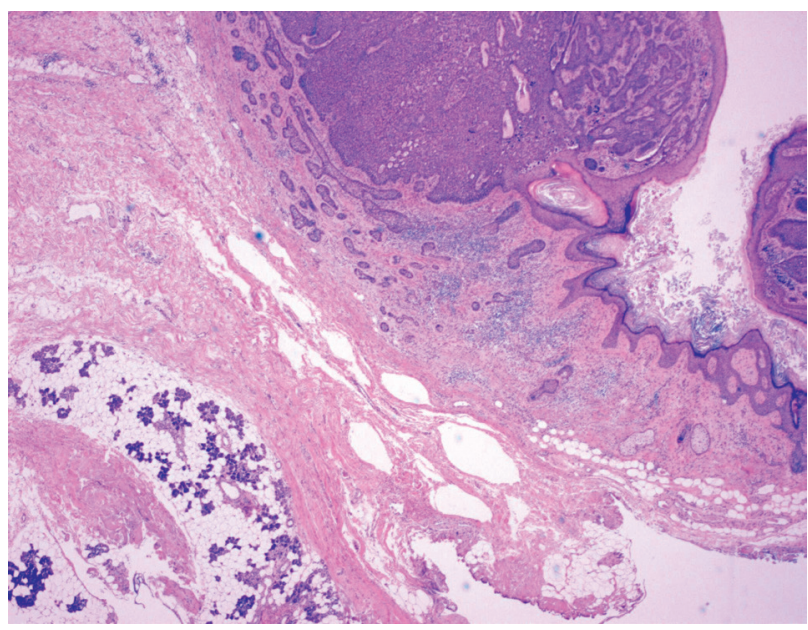

Fig. 4. Case 1. Strands of basal cell carcinoma (BCC) are directly adhered to the parotid gland, and extend to the parotid tissue located at the left bottom of the photograph $(\mathrm{H} \& \mathrm{E}, \times 20)$. ly removed with MMS, in which wide excision using a fasciocutaneous advancement flap, split-thickness skin graft, and a local flap were employed. All lesions were completely resected by MMS and wide excision, and they were covered with a fasciocutaneous advancement flap and a split-thickness skin graft. Pathologic evaluation confirmed the diagnosis of BCC. Only the abdominal lesion was classified as Stage II, and other lesions were classified as Stage I.

\section{DISCUSSION}

BCC is the most common human cancer that usually occurs as a single lesion, mostly on the face and neck. Multiple BCCs are not uncommon as there is a $36 \%-50 \%$ increased risk of development of additional BCCs after the first lesion within 5 years [7]. The literature is quite variable with respect to the recurrence rates of these tumors, and it reports recurrence rates between $10 \%$ and $67 \%$. Recurrence rates between $5 \%$ and $14 \%$ are evident when tumors are completely excised [8].

The most significant risk factor involved in the pathogenesis is ultraviolet light which triggers mutations in tumor suppressor genes [1]. Other factors include exposure to ionizing radiation, arsenic, and polyaromatic hydrocarbons, which appear to be involved in mutations of regulatory genes and alterations in immune surveillance. Increasing age, fair skin types I and II, male sex, immunosuppression, and arsenic exposure are the other established risk factors. Inherited conditions such as the nevoid BCC syndrome (Gorlin syndrome), Rombo syndrome, unilateral basal cell nevus syndrome, Bazex syndrome are common inherited conditions with multiple BCC manifestations $[1,2]$. The occurrence of multiple BCCs has been reported in many literatures, but our cases were quite different from these cases. History and clinical examination were not consistent with Gorlin syndrome. Nonsyndromic, but hereditary multiple BCCs have been reported in literatures, but they were ruled out based on the negative family history [9]. There was no history of exposure to arsenic, irradiation, dry ice and no evidence of keratoacanthoma or xeroderma pigmentosum [10,11]. Cytochrome P450 (CYP2D6) genotypes and Glutathione S transferase (GSTM1 and GSTT1) are included with a multiple presentation phenotypes of BCC and have been 

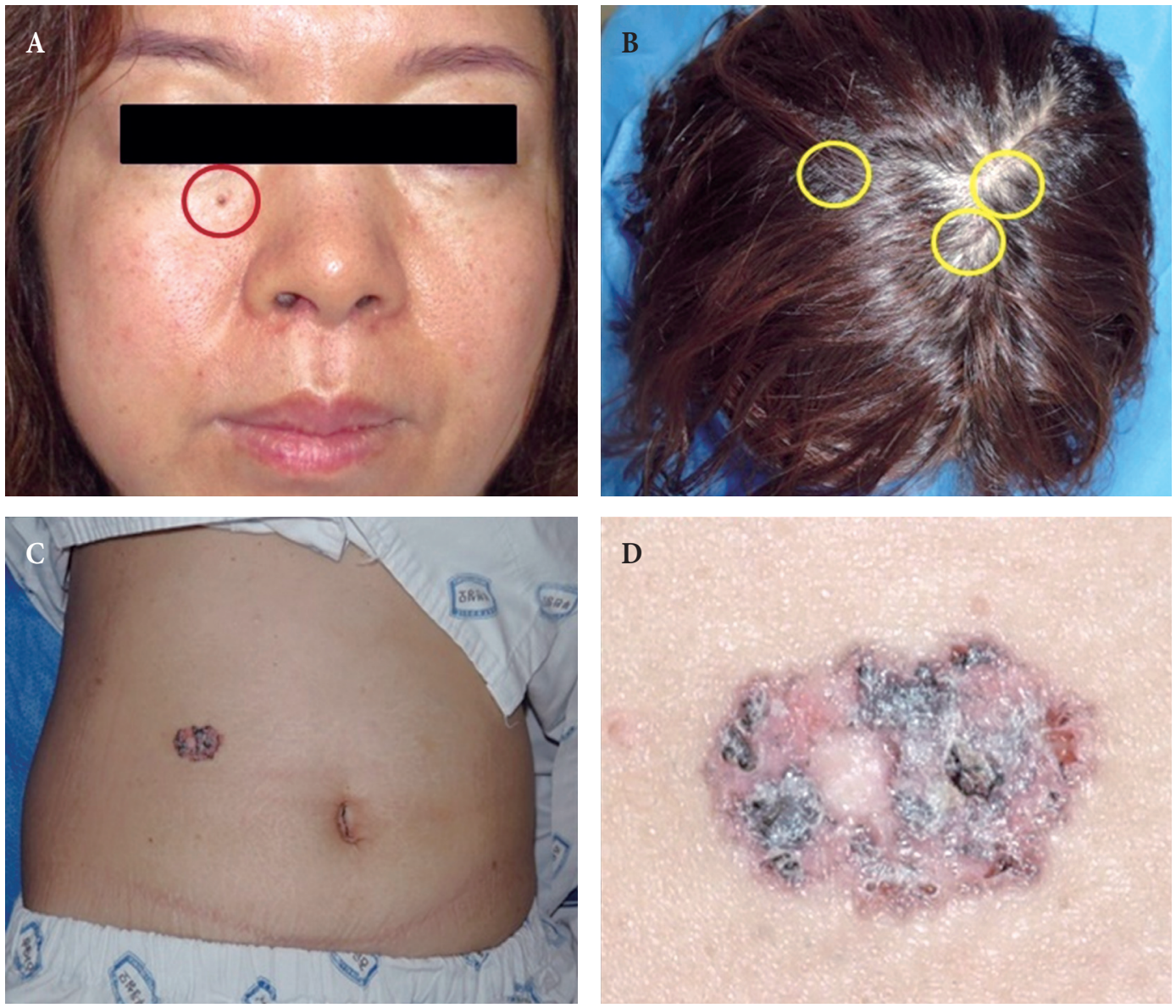

Fig. 5. Case 2. Preoperative photography shows that the lesions were located on the (A) face (red circle), (B) scalp (yellow circle), and (C, D) abdomen.

introduced in the literature [12]. However, considering the financial status of the patients, we decided not to perform Polymerase chain reaction (PCR) assay to rule out this genotype. Moreover, the treatment plan would not be affected by the PCR result. Hence, we categorized our cases as nonsyndromic and nonhereditary type of multiple basal cell carcinomas worth mentioning.

Furthermore, Case 1 showed parotid invasion of the cancer, which is found in only $1 \%$ to $1.5 \%$ of all cutaneous cancers of the head and neck [13]. Christensen et al. [6] reviewed treatment and outcomes of patients with BCC involving the parotid gland by direct infiltration, and they emphasized that recurrent BCC involving the parotid gland through direct infiltration has a high rate of future recurrence, and adjuvant treatment may be required. Our patient had previously received surgical removal of BCC in the right posterior auricular area, and medical reports showed that the lesion was small in size without parotid invasion. However, direct extension to the parotid gland may not have been detected and this could have possibly caused recurrence of cancer as adjuvant therapy was not administered. MMS or wide local excision (WLE) with intraoperative margin control is accepted as a reasonable first-line treatment for primary BCC involving the parotid gland [6]. Thus, we performed WLE with partial parotidectomy along with intraoperative histological assessment of surgical margins of the tumor in the right posterior auricular area and MMS for other tumors. The treatment guidelines published by the British Association of Dermatologists and the American Academy of Dermatology aim for eradication of the tumor with an acceptable cosmetic outcome for the patient. Surgical excision with intraoperative margin assessment is considered to have the lowest overall failure rate in BCC treatment. 
Nonsurgical techniques such as cryosurgery, radiation therapy, photodynamic therapy, and curettage do not allow histological confirmation of tumor clearance and they are usually used to treat low-risk tumors, although radiation therapy plays an important role in the management of high-risk BCC [14].

Recently, it has been discovered that the Hedgehog pathway has an important role in the pathogenesis of BCC. Most BCCs have mutations in the gene that is responsible for the signaling pathway, called Hedgehog. The Hedgehog pathway is crucial for the development of the embryo and fetus, and it plays an important role in some adult cells. However, this pathway may be overexpressed in BCC cells, and targeted drugs have been developed. Vismodegib has recently been approved by the US Food and Drug Administration in patients with advanced BCC [15]. Cowey [15] stated that the use of Vismodegib should be considered in patients with locally advanced or metastatic BCC which has recurred despite two or more surgical resections or when there are too many lesions to be resected. Our patients did not fulfill the criteria, but we can consider using Vismodegib when we experience a recurrent case or an unresectable case of BCC.

$\mathrm{BCC}$ is rarely metastatic, but it can lead to severe disfiguration or destruction. It is important to diagnose and treat BCC at an early stage. We encountered two cases of nonsyndromic and nonhereditary type of multiple BCCs, and one of these cases is a very rare case with parotid invasion. MMS or WLE with intraoperative margin assessment is recommended as first-line treatment in cases of multiple BCCs. Also, the same approach can be applied to lesions with parotid invasion. If surgical resection is not feasible, pharmacological treatment may be considered.

\section{PATIENT CONSENT}

The patient provided written informed consent for the publication and the use of their images.

\section{REFERENCES}

1. Zak-Prelich M, Narbutt J, Sysa-Jedrzejowska A. Environmental risk factors predisposing to the development of basal cell carcinoma. Dermatol Surg 2004;30(2 Pt 2):248-52.

2. McNaughton SA, Marks GC, Green AC. Role of dietary factors in the development of basal cell cancer and squamous cell cancer of the skin. Cancer Epidemiol Biomarkers Prev 2005;14:1596-607.

3. Alghamdi Y. Skin tags as a presenting sign of basal cell nevus syndrome in three sisters of the same family. Ann Saudi Med 2008;28:132-4.

4. Lu Y, Zhu HG, Ye WM, Zhang MB, He D, Chen WT. A new mutation of PTCH gene in a Chinese family with nevoid basal cell carcinoma syndrome. Chin Med J (Engl) 2008;121:118-21.

5. Farley RL, Manolidis S, Ratner D. Aggressive basal cell carcinoma with invasion of the parotid gland, facial nerve, and temporal bone. Dermatol Surg 2006;32:307-15.

6. Christensen KN, Henderson GP, Hocker TL, Otley CC, Roenigk RK. Outcomes of basal cell carcinomas directly invading the parotid gland. Dermatol Surg 2015;41:390-6.

7. Robinson JK. Risk of developing another basal cell carcinoma: a 5-year prospective study. Cancer 1987;60:118-20.

8. Lara F, Santamaria JR, Garbers LE. Recurrence rate of basal cell carcinoma with positive histopathological margins and related risk factors. An Bras Dermatol 2017;92:58-62.

9. Happle R. Nonsyndromic type of hereditary multiple basal cell carcinoma. Am J Med Genet 2000;95:161-3.

10. Ahn SK, Lee HS, Han SK, Lee SH, Lee S. Multiple basal cell carcinoma associated with keratoacanthoma. Yonsei Med J 1992;33:277-80.

11. Malhotra AK, Gupta S, Khaitan BK, Verma KK. Multiple basal cell carcinomas in xeroderma pigmentosum treated with imiquimod 5\% cream. Pediatr Dermatol 2008;25:488-91.

12. Ramachandran S, Lear JT, Ramsay H, Smith AG, Bowers B, Hutchinson PE, et al. Presentation with multiple cutaneous basal cell carcinomas: association of glutathione S-transferase and cytochrome P450 genotypes with clinical phenotype. Cancer Epidemiol Biomarkers Prev 1999;8:61-7.

13. Maydan E, Desciak EB, Eliezri YD. Management of cutaneous neoplasms invading the parotid gland. Dermatol Surg 2006;32:1209-13.

14. Bath-Hextall FJ, Perkins W, Bong J, Williams HC. Interventions for basal cell carcinoma of the skin. Cochrane Database Syst Rev 2007;(1):CD003412.

15. Cowey CL. Targeted therapy for advanced Basal-cell carcinoma: vismodegib and beyond. Dermatol Ther (Heidelb) 2013;3:17-31. 